\title{
«Io sostituisco i cereali!» Origine e primato della palma nelle culture dell'antica Mesopotamia
}

\author{
Lorenzo Verderame \\ Dipartimento Istituto Italiano di Studi Orientali, Sapienza - Università di Roma, IT \\ lorenzo.verderame@uniroma1.it \\ <http://lorenzoverderame.site.uniroma1.it/>
}

\begin{abstract}
In this paper I discuss various aspects related to the imaginary and to the symbolism of the date palm in the written sources from ancient Mesopotamia through the analysis of two compositions. The first is a Sumerian text known as Inanna and Šukaletuda, which contains in the first part a myth describing the creation of the date palm by a raven instructed by the god Enki. The second is a Babylonian dispute, Palm and Tamarisk, in which the two trees try to prevail one over the other, proclaiming their virtues and functions. The analysis shows that, although prerogatives and associations are shared by other trees, the palm holds the primacy over all of them. In the conclusions, I argue that one of the possible reasons of such preeminence is that the palm is the only one of these trees that produces a fruit - the date - which, moreover, is on the economic and cultural level is equal to the primary cultivation of cereals.
\end{abstract}

\section{Keywords}

Mesopotamia; Sumer; Sumerian and Akkadian literature; Debate poems; Mythology; Date palm; Tamarisk; Poplar; Cedar

\section{Sommario/Content}

o. Introduzione

1. La palma e la Mesopotamia meridionale

2. La creazione della palma da dattero: Inanna e Šukaletuda

3. Palma e Tamarisco

4. Conclusioni

Bibliografia 


\section{Ocula ${ }^{23}$}

Vol 21, No 23 (July 2020) • DOI: 10.12977/ocula2020-26

Lorenzo Verderame | «Io sostituisco i cereali!». Origine e primato della palma nelle culture

dell'antica Mesopotamia

\section{o. Introduzione}

Nel presente contributo presento un'analisi degli aspetti simbolici del principale albero dell'antica Mesopotamia, la palma da dattero. Nonostante la rilevanza di questa coltura tanto sul piano economico quanto su quello culturale, non esiste una trattazione dell'immagine della palma nelle tradizioni mesopotamiche. ${ }^{1}$ Diversi studi sono stati dedicati ad aspetti specifici di quest'albero come quello lessicografico di Landsberger (1967), quello sulla coltura nei documenti economici del periodo paleo-babilonese di Coquerillat (1967) o quello sull'iconografia della Danthine (1937), ma, oltre a essere datati, non si occupano degli aspetti simbolici della palma nelle culture mesopotamiche. Accenni a questi ultimi si trovano in forma puntuale o generale nei commenti all'edizione di testi letterari che menzionano la palma o allo studio dell'iconografia della stessa. ${ }^{2}$

Non è mia intenzione esaurire tutti gli aspetti di questo argomento nel presente contributo, ma presentare una panoramica generale della simbologia della palma e, per contrasto, di alcuni altri importanti alberi della tradizione mesopotamica (tamarisco, pioppo e albero eren, tradizionalmente identificato con il cedro).

Oggetto principale dello studio e punto di partenza per ulteriori riflessioni sono due testi letterari, Inanna e Šukaletuda e Palma e Tamarisco. Si tratta di composizioni che appartengono a due tradizioni differenti, essendo il primo un mito in lingua sumerica e il secondo una tenzone in lingua accadica. Sebbene i testimoni delle due composizioni siano in parte coevi, ${ }^{3}$ la composizione sumerica affonda le sue radici nella tradizione mitografica del III millennio, mentre la composizione babilonese appartiene alla letteratura sapienziale in lingua accadica del II millennio. Nonostante queste differenze, i due testi presentano una comunanza di motivi che va interpretata alla luce della centralità della palma nelle culture mesopotamiche e della trasmissione letteraria nell'ininterrotta tradizione cuneiforme. ${ }^{4}$

\section{La palma e la Mesopotamia meridionale}

La palma da dattero è l'albero che l'immaginario occidentale associa al Vicino Oriente. Tale impressione dettata da una romantica visione "orientalistica" riflette e trova conferma nella rilevanza che questa coltura ha nella regione

1 Kalla (2018: 869 e n. 29).

2 Volk (1995); Streck (2004); Howard-Carter (1982); Seidl e Sallaberger (2006); Kalla (2018).

3 I testimoni di Inanna e Šukaletuda provengono in massima parte dai livelli paleo-babilonesi (ca. XVIII sec.) dei quartieri scribali della città di Nippur; allo stesso periodo risale la più antica testimonianza di Palma e Tamarisco, rinvenuta nell'antica città di Šaduppûm (mod. Tell Ḥarmal).

4 Per la continuità dei motivi legati alla palma oltre la tradizione mesopotamica vd. n. 50 e, per l’iconografia, Kalla (2018). 


\section{Ocula ${ }^{23}$}

Vol 21, No 23 (July 2020) • DOI: 10.12977/ocula2020-26

Lorenzo Verderame | «Io sostituisco i cereali!». Origine e primato della palma nelle culture dell'antica Mesopotamia

da oltre cinquemila anni (figg. 1-2). ${ }^{5}$ Una tradizione ininterrotta che ha portato l'UNESCO a inserire nel 2019 la palma da dattero e la sua coltivazione nella lista del Patrimonio dell'umanità (World Heritage). A tutt'oggi la palma da dattero con le sue numerose varietà è una delle principali colture dell'Iraq che è stato a lungo il maggiore produttore di datteri. ${ }^{6}$

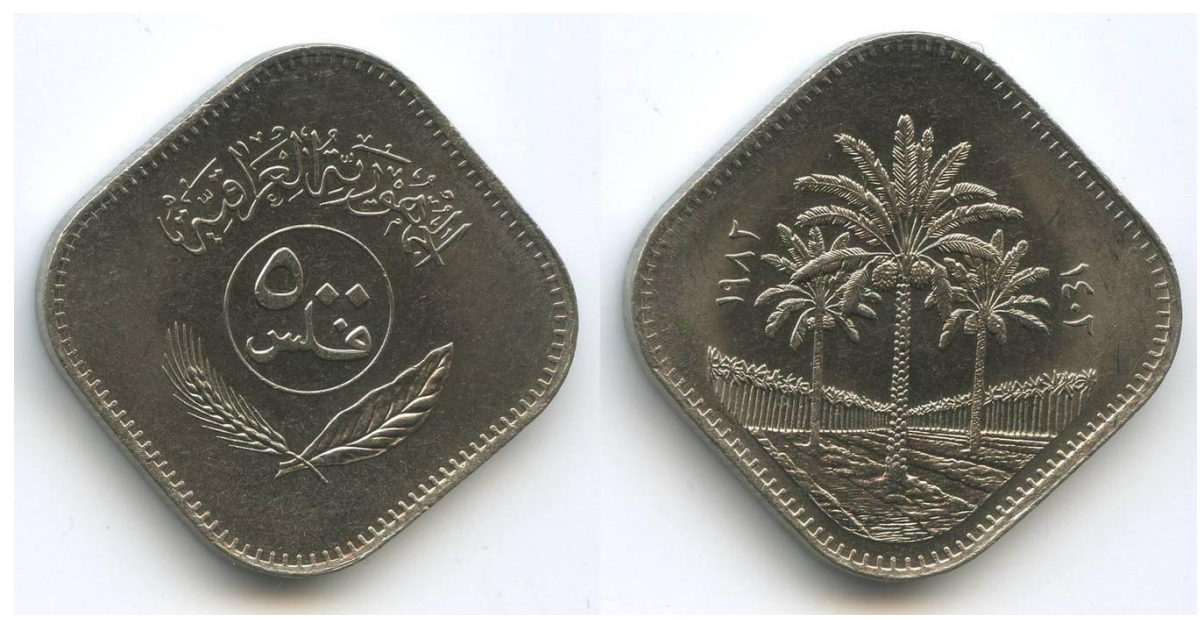

Figura 1. Moneta irachena da 500 fils.

Nell'antica Mesopotamia la coltivazione della palma da dattero ${ }^{7}$ costituisce uno dei due pilastri dell'economia agraria della regione assieme a quella dei cereali. La palma e l'orzo si collocano ai due poli opposti dell'attività agricola e generano differenti associazioni, ${ }^{8}$ ma il loro valore simbolico ed economico è posto sullo stesso piano, come sottolineano i passi tratti dalle due composizioni analizzate in questo studio. In Inanna e Šukaletuda, descrivendo la palma, si dice che «i suoi datteri sono posti a lato dell'orzo spulato» (l. 83).

5 Per una introduzione generale alla letteratura sumerica e accadica vd. Verderame (2016), cui si rimanda per una bibliografia delle singole opere. Per un'antologia in italiano dei testi mitologici vd. Bottéro e Kramer (1992) e Pettinato (2001); per i testi letterari non mitologici vd. Castellino (1977). Gran parte della letteratura sumerica è disponibile in trascrizione e traduzione inglese sul sito del progetto Electronic Text Corpus of Sumerian Literature (= eTCSL; <http://etcsl.orinst.ox.ac.uk/>); per la letteratura accadica del III e II millennio si veda il progetto Sources of Early Akkadian Literature (SEAL; <https://seal.huji.ac.il>).

6 Vd. la recente rassegna di Zabar e Borowy (2012).

7 Sum. ĝišnimbar/ĝišimmar, acc. gišimmaru. Per la palma nell'antica Mesopotamia si veda in generale Streck (2004: 263-274; 2012) con bibliografia precedente.

8 In generale, si può notare come alla "semplicità" e diffusione della coltura dei cereali si opponga quella localizzata e tecnica della palma da dattero. All'agricoltore che semina il chicco di cereale si oppone l'esperto giardiniere che deve procedere all'impollinazione manuale delle infiorescenze maschili con quelle femminili nelle palme. 


\section{Ocula ${ }^{23}$}

Vol 21, No 23 (July 2020) • DOI: 10.12977/ocula2020-26

Lorenzo Verderame | «Io sostituisco i cereali!». Origine e primato della palma nelle culture dell'antica Mesopotamia

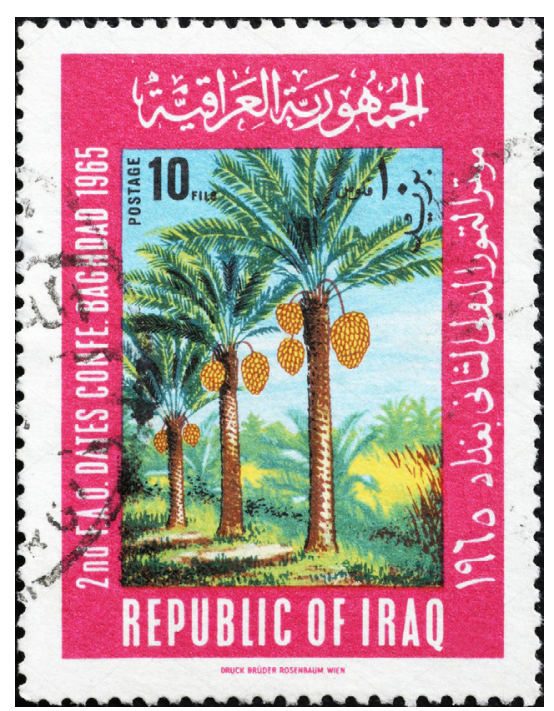

Figura 2. Francobollo iracheno commemorativo della seconda conferenza sui datteri organizzata dalla F.A.O. a Baghdad (1965).

Nella tenzone con Tamarisco, Palma esalta il suo frutto e sostiene addirittura «Io sostituisco i cereali!» (Palma e Tamarisco Ab 46'). Al di là degli aspetti letterari e simbolici, i numerosi testi amministrativi confermano tanto l'attenzione e le energie profuse dalle amministrazioni centrali per la coltivazione della palma, quanto la meticolosa registrazione in entrata e in uscita di datteri e di altri prodotti da essi derivati, confermando su un piano di fonti documentarie la rilevanza della coltura della palma che si pone sullo stesso piano dell'allevamento e dei cereali. Il re Sargon II d'Assiria (VIII sec. a.C.), provenendo dal nord e abituato a un altro tipo di colture, conferma la rilevanza della palma per l'economia del sud mesopotamico, quando dichiara: «Ho tagliato

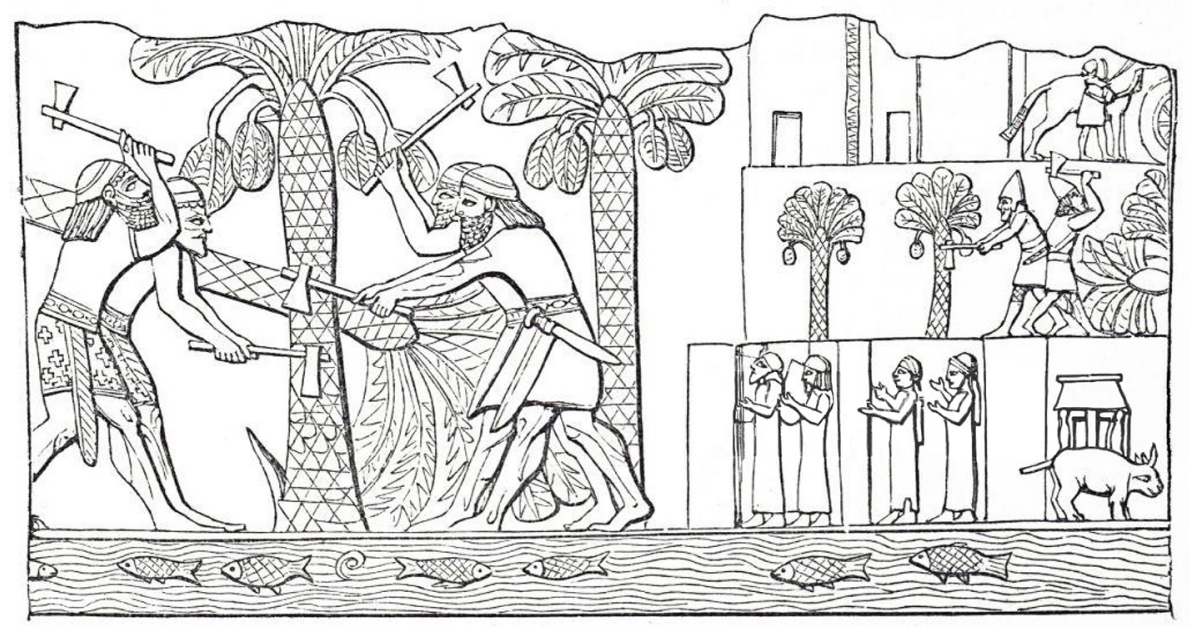

Figura 3. Soldati assiri abbattono delle palme nella Mesopotamia meridionale, particolare da un disegno di un rilievo assiro dal palazzo sud-ovest di Ninive del periodo di Sennacherib. 


\section{Ocula ${ }^{23}$}

Vol 21, No 23 (July 2020) • DOI: 10.12977/ocula2020-26

Lorenzo Verderame | «Io sostituisco i cereali!». Origine e primato della palma nelle culture dell'antica Mesopotamia

le palme, che sono il loro sostentamento, e i giardini che sono l'ornamento del paese» (cf. fig. 3). ${ }^{9}$

Nei rilievi assiri del I millennio diffuso è l'uso della palma quale elemento caratteristico del paesaggio della Mesopotamia meridionale (fig. 4). ${ }^{10}$

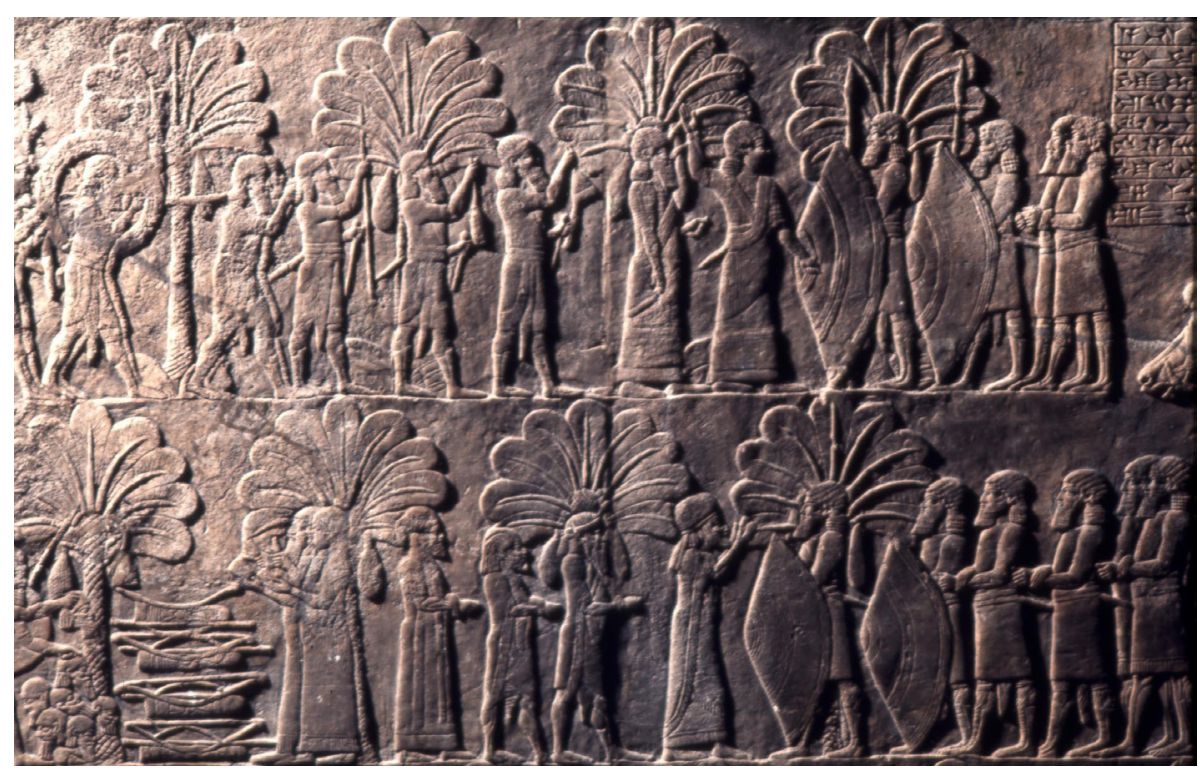

Figura 4. Particolare di rilievo assiro con rassegna del bottino dopo la conquista di Babilonia da parte di Assurbanipal (British Museum, BM 124946).

La palma è un motivo iconografico diffuso fin dalle epoche più antiche e si ritrova in diverse espressioni delle arti plastiche. Come nei rilievi, anche nei sigilli la palma è impiegata per caratterizzare un ambiente o quale elemento di separazione o cornice delle scene. Alla tradizionale iconografia della palma, si aggiunge a partire dalla metà del II millennio a.C. un motivo noto come "palmetta", la quale, tuttavia, sembra avere un'origine occidentale. ${ }^{11}$ Uno dei pochi affreschi conservati del Vicino Oriente antico, la cosiddetta scena di intronizzazione di Zimri-Lim (XVIII sec a.C.) dal palazzo reale di Mari (Tell Hariri), ${ }^{12}$ presenta un'articolata composizione ripartita in diversi registri incorniciati da due alberi di palma, dietro i quali, specularmente due divinità benedicono la scena (fig. 5). Sulle palme sono rappresentante due scimmie che si arrampi-

9 Fuchs (1994: 330 1l. 289-290).

10 Galter (1989). Nonostante l'associazione della palma con il sud sumerico, si è voluto identificare con essa il cosiddetto "albero sacro" assiro, una stilizzazione di un albero simbolico ai cui lati sono spesso simmetricamente rappresentanti geni o lo stesso sovrano; per l'“albero sacro" assiro vd. in generale Giovino (2007).

11 Cf. Seidl e Sallaberger (2006: 54-61).

12 L'affresco è oggi conservato presso il museo del Louvre. Un'immagine dell'affresco è disponibile sul sito del museo (<https://www.louvre.fr/en/oeuvrenotices/mural-painting >). 


\section{Dcula ${ }^{23}$}

Vol 21, No 23 (July 2020) • DOI: 10.12977/ocula2020-26

Lorenzo Verderame | «Io sostituisco i cereali!». Origine e primato della palma nelle culture dell'antica Mesopotamia

cano. Una sezione speculare è delimitata dalla palma e da un altro albero e lo spazio è diviso in tre registri, in cui troviamo, fronteggiandosi, a destra e sinistra, due esseri alati dal corpo leonino (forse una sfinge e un grifone) e un toro (zebù) androcefalo che poggia una zampa su una montagna. Al centro vi è la scena dell'investitura, inserita in una cornice che forse riproduce il palazzo reale. La scena è divisa in due registri. Quello inferiore è occupato da due divinità speculari con vaso zampillante (aryballos). Quello superiore rappresenta al centro il re che riceve dalla dea Ištar l'anello e la bacchetta, simbolo del potere e della regalità; alle loro spalle due divinità tutelari.

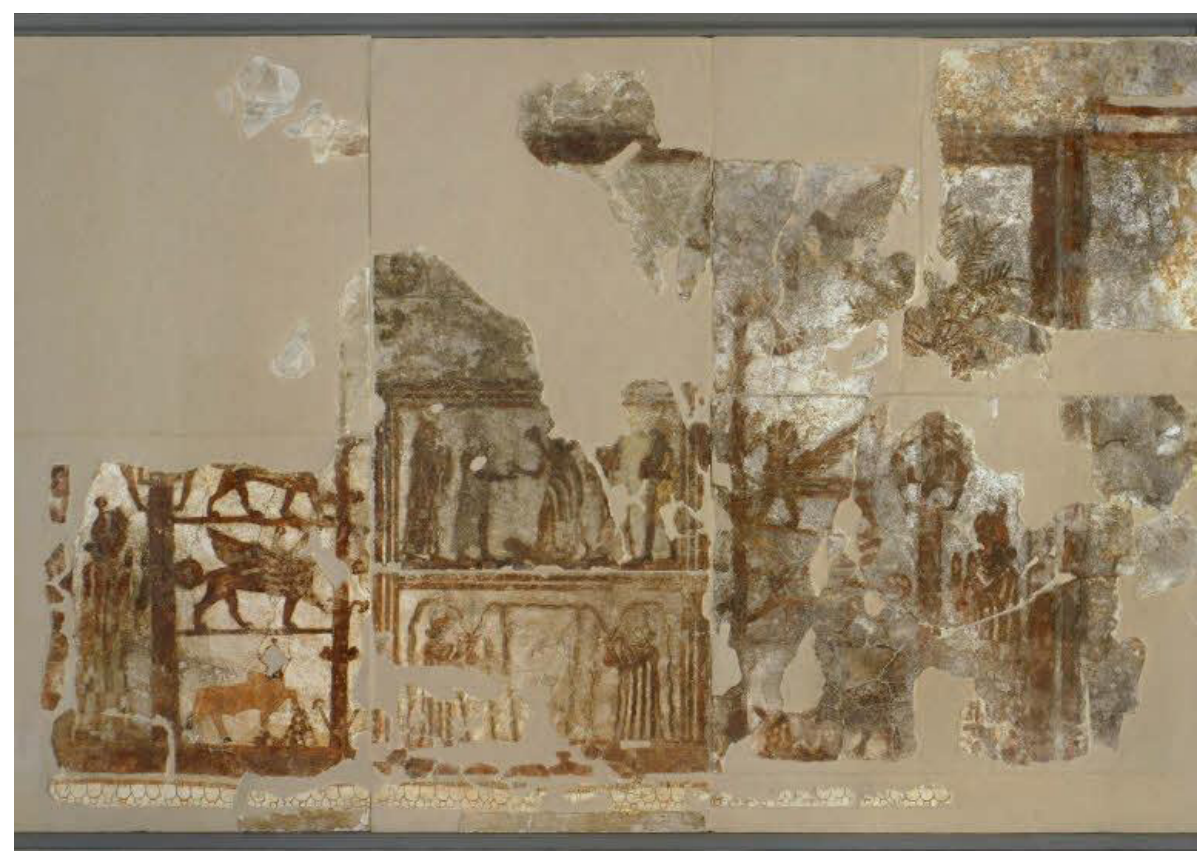

Figura 5. Affresco con la cosiddetta "scena dell'investitura" di Zimri-Lim (XIX sec. a.C.) dal palazzo di Mari (Museo del Louvre, AO 19826).

La palma è ampiamente usata anche nella decorazione architettonica. Interessante è il peculiare uso di mattoni modanati in forma di palma da dattero per decorare i muri di templi e palazzi. ${ }^{13}$ Privi di materie prime di qualche valore, gli artigiani mesopotamici si sono ingegnati a creare partendo dall'unico materiale a loro disposizione in abbondanza, l'argilla. Nicchie e lesene sono state utilizzate per creare ombre, così come i mattoni modanati e, più tardi, i mattoni invetriati per creare decorazioni. Si diffonde nell'architettura del periodo paleo-babilonese la decorazione delle facciate di edifici con colonne d'argilla a tortiglione che riproducono il fusto della palma da dattero (fig. 6). ${ }^{14}$ Il caso più interessante in tal senso è quello del tempio di Tell

13 Moorey (1999: 309-312).

14 Moorey (1994: 310-312). 


\section{Ocula ${ }^{23}$}

Vol 21, No 23 (July 2020) • DOI: 10.12977/ocula2020-26

Lorenzo Verderame | «Io sostituisco i cereali!». Origine e primato della palma nelle culture dell'antica Mesopotamia

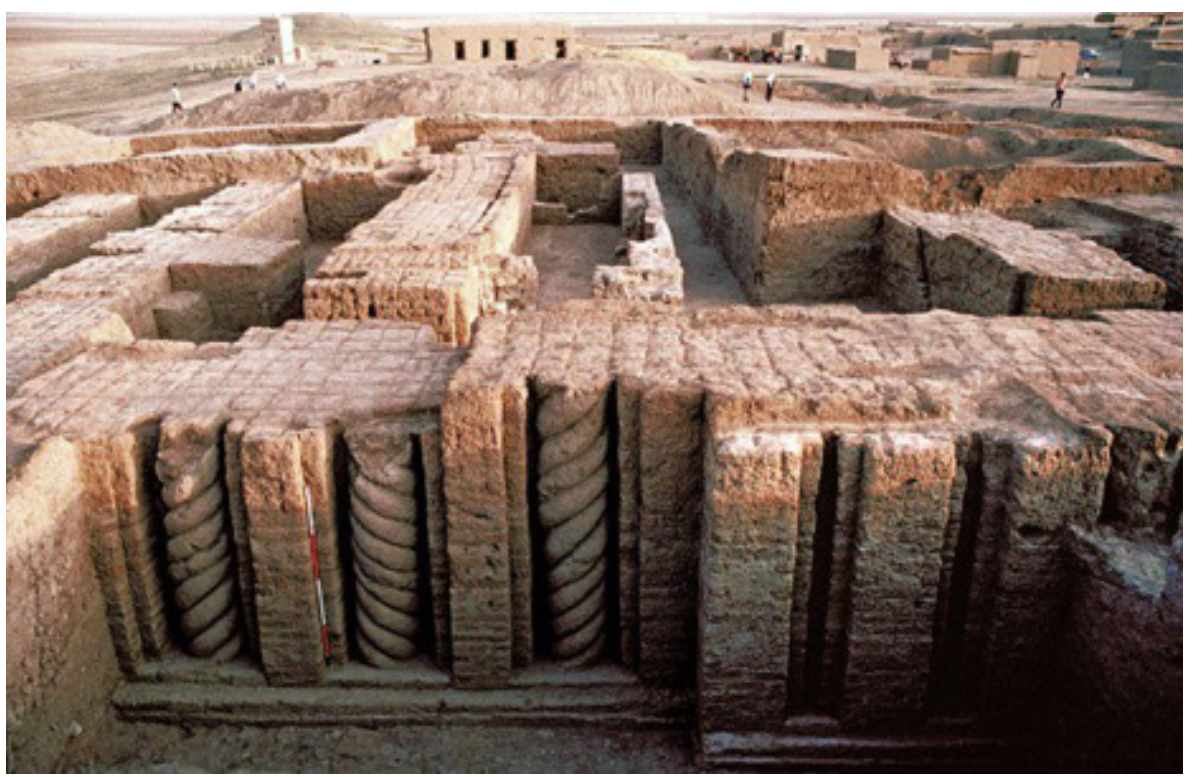

Figura 6. Decorazione della facciata del tempio nord-est dell'acropoli di Tell Leilan (XVIII sec. a.C.).

al-Rimah risalente al XVIII secolo a.C., dove troviamo mattoni modanati che riproducono palme con fusti a tortiglione utilizzati per decorare la facciata esterna del tempio, cui fa da pendant un programma decorativo di rilievi di pietra dove diverse figure divine e esseri compositi sono incorniciati tra due palme (fig. 7). ${ }^{15}$

\subsection{Un albero autoctono? La palma di Dilmun}

Nonostante la grande importanza ricoperta dalla palma nelle culture mesopotamiche, le fonti più antiche insistono sull'origine straniera di quest'albero. In particolare, la palma da dattero è associata con Dilmun.

Nel prato dove ho eretto gli altari per il mio santuario di Ur ho davvero fatto crescere le palme come nel paese di Dilmun. (Nanna I 34-35 // 38-39)

Dilmun è una delle tre principali entità politico-geografiche con cui il sud sumerico intrattiene rapporti commerciali e culturali nel Golfo Persico nel III millennio a.C. Le altre due sono Magan e Meluhha, identificate rispettivamente con l'Oman e la regione costiera compresa tra Pakistan e India del Nord.

Quanto a Dilmun, la sua identificazione sembra essere di gran lunga più sicura grazie soprattutto agli sviluppi dell'archeologia nell'area del golfo degli ultimi trent'anni. Dilmun sarebbe da identificare con l'attuale Bahrein, dove sono presenti migliaia di tumuli contemporanei delle culture mesopotamiche.

15 Howard-Carter (1982). 


\section{Ocula ${ }^{23}$}

Vol 21, No 23 (July 2020) • DOI: 10.12977/ocula2020-26

Lorenzo Verderame | «Io sostituisco i cereali!». Origine e primato della palma nelle culture dell'antica Mesopotamia

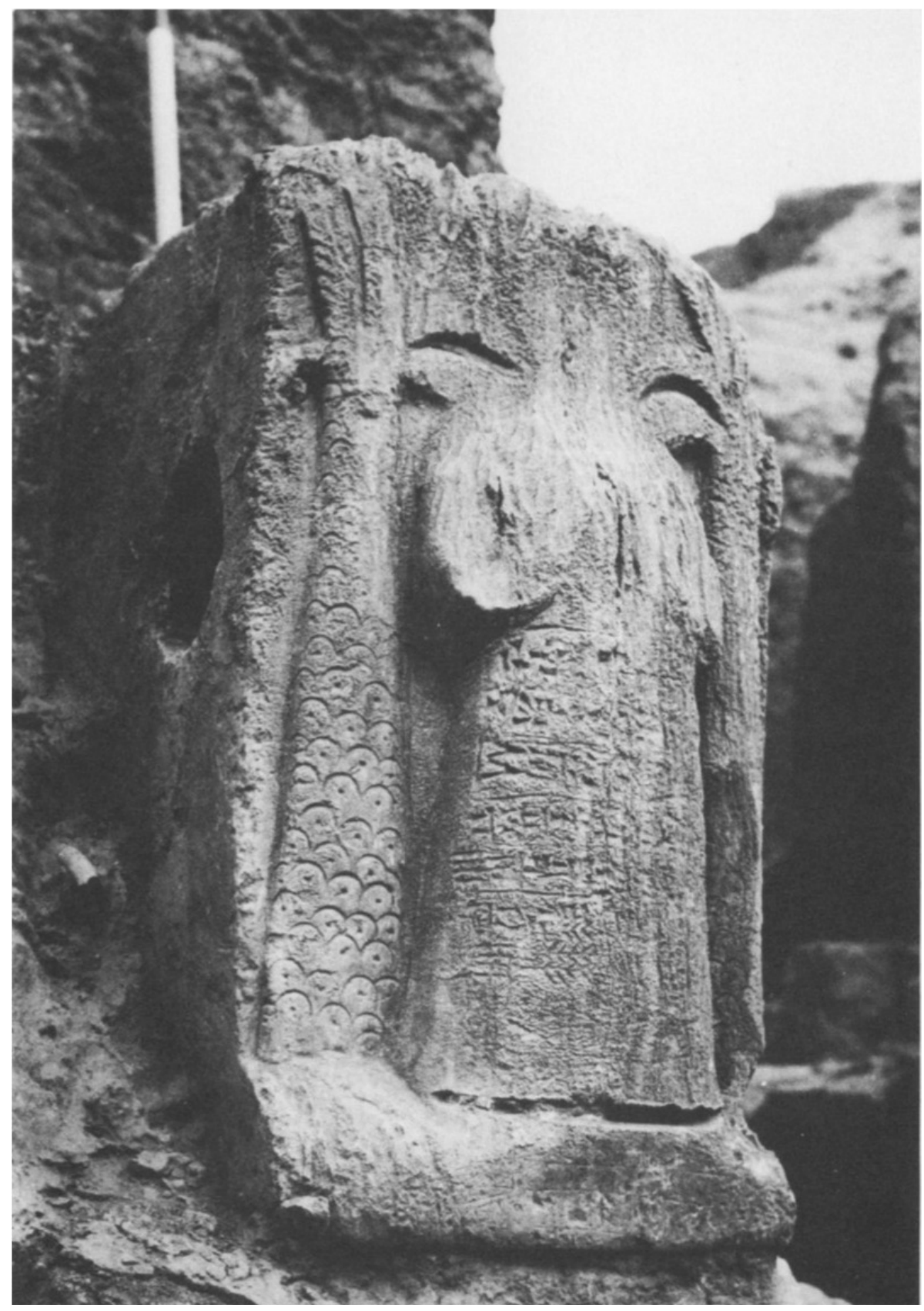

Figura 7. Particolare dell'apparato decorativo della facciata del tempio di Tell al-Rimah con la "Signora tra le palme" (da Howard-Carter 1982).

Un mito sumerico (Enki e Ninhursaĝa) narra della creazione o, meglio, della trasformazione di Dilmun da luogo "selvaggio" ad ambiente urbano civilizzato. Le vicende si svolgono in un'età mitica e primeva, sottolineando l'antichità tributata in altre fonti a Dilmun, il cui nome diviene sinonimo stesso di arcaicità e la cui realtà si confonde con il mito. In tal senso non sorprende che il parallelo con la palma e Dilmun sia rievocato per sostanziare un'ancora 


\section{Ocula ${ }^{23}$}

Vol 21, No 23 (July 2020) • DOI: 10.12977/ocula2020-26

Lorenzo Verderame | «Io sostituisco i cereali!». Origine e primato della palma nelle culture dell'antica Mesopotamia

più arcaica origine di una determinata città, come nel caso di Nippur e Isin nei passi sotto riportati:

La mia città, quando Dilmun non esisteva ancora, faceva crescere le palme! Nippur, quando Dilmun non esisteva ancora, faceva crescere le palme!

(Viaggio di Nanna-Su'en a Nippur 34-35)

La mia casa, quando Dilmun non esisteva ancora, fu generata da una palma! Isin, quando Dilmun non esisteva ancora, fu generata da una palma!

(Ninisina A 93-94)

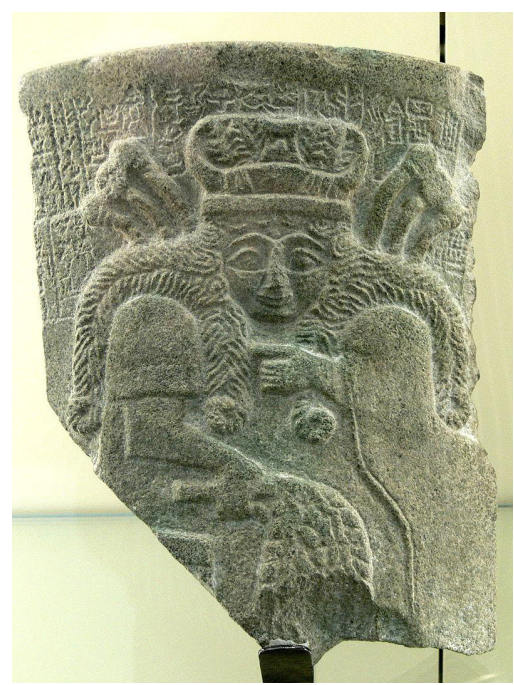

Figura 8. Frammento di vaso di clorite con iscrizione del re (ensi) di Lagaš, Enmetena (ca. XXV sec. a.C.), e raffigurazione a rilievo di dea (forse BaU) assisa in trono con un ramo di datteri in mano (Vorderasiatisches Museum di Berlino, VAT 16438).

\section{La creazione della palma da dattero: Inanna e Šukaletuda}

Nella tradizione sumerica, la palma non è un dono divino disceso dal cielo né la conseguenza di un'agricoltura che scaturisce direttamente dalla creazione e mantenimento dei canali, come nel caso dei cereali. Un mito sumerico narra della creazione della palma da dattero e della sua coltivazione. La composizione è nota come Inanna e Šukaletuda, titolo attribuitogli dai moderni studiosi, ${ }^{16}$ mentre era nota nell'antichità in base al suo incipit, Signora dai grandi $M E$ (in-nin me-gal-gal-la), che si ritrova in diversi cataloghi di opere sumeriche. Il testo è composto di due unità narrative. Nella prima è descritto come il dio Enki insegni a Corvo a coltivare la palma da dattero. Nella seconda

16 L’edizione scientifica del testo è a opera di Volk (1995); cf. eTCSL 1.3.3; traduzioni italiane si trovano in Bottéro e Kramer (1992: 266-282) e Pettinato (2001: 380-395). Per l'interpretazione del testo vd. anche Selz (2001) e Mařik (2003). 


\section{Ocula ${ }^{23}$}

Vol 21, No 23 (July 2020) • DOI: 10.12977/ocula2020-26

Lorenzo Verderame | «Io sostituisco i cereali!». Origine e primato della palma nelle culture dell'antica Mesopotamia

parte, Šukaletuda, il giardiniere, ${ }^{17}$ abusa sessualmente della dea Inanna ${ }^{18}$ che si è fermata a riposare nel suo giardino. La dea, irata, cerca in diversi modi di scoprire il colpevole, ma Šukaletuda riesce a sfuggire grazie all'aiuto di Enki. Infine, Inanna si rivolge proprio a quest'ultimo che non potrà rifiutarsi di consegnare Šukaletuda. La dea deve punire Šukaletuda con la morte, ma gli attribuirà una fama imperitura. ${ }^{19}$

L'intera prima parte contiene la storia del corvo o di Corvo (uga ${ }^{\text {mušen }}$ ), ${ }^{20}$ che, istruito dal dio Enki, darà vita alla coltivazione della palma da dattero. Dopo un'introduzione dove è descritta la preparazione della dea Inanna che si appresta a partire ${ }^{21}$ e una lacuna di una ventina di righe, il testo riprende con il dio Enki che sta impartendo le istruzioni a Corvo:

Corvo, ti darò delle istruzioni! Alle [mie] istruzioni [presta attenzione!] Corvo, nel santuario ti darò delle istruzioni! Alle [mie] istruzioni [presta attenzione!] Il kohl dell'arte dell'esorcismo di Eridu con l'ascia ... e mastica assieme a olio/grasso e acqua in una ciotola di lapislazzuli posta in una stanza dell'agrun. Poi pianta(lo in?) un terreno acquitrinoso per porri in un orto da verdura. Allora sradica [...]. (Inanna e Šukaletuda 49-56)

A questo punto Corvo, eseguendo alla lettera le istruzioni del suo signore, il dio Enki, si mette all'opera. Macina il kohl dell'esorcismo di Eridu, lo mastica e mischia con acqua e olio in una ciotola che viene poi riposta nella sacrestia del santuario. Dopodiché lo pianta in un campo per ortaggi, ma in un terreno acquitrinoso adatto alla coltivazione dei porri. ${ }^{22}$ Il risultato è straordinario.

17 Per il Corvo, la sua relazione con Šukaletuda e il destino di quest'ultimo, da associare al giardiniere Išullanu trasformato in rospo menzionato nell'Epopea di Gilgameš (VI 64-79), vd. Volk (1995: 53-64) e Verderame (in stampa). Per la figura del giardiniere nella letteratura mesopotamica vd. Besnier (2002) e Rendu Loisel (2013).

18 Non è chiaro il tipo di relazione che intercorre tra i vari elementi delle due narrazioni. Se è possibile ipotizzare un legame tra Corvo e Šukaletuda (vd. n. 17), difficile è dire quanto abbia a che fare la creazione della palma con la vicenda di Inanna. La presunta relazione tra la dea e la palma da dattero ha portato Jacobsen (1957: 107-109 n. 32) a proporre la lettura del nome della dea quale "signora dei datteri". Per quanto occasionalmente ripresa, per es. in Howard-Carter (1982), tale interpretazione non ha mai avuto un seguito negli studi assiriologici. Per varie ragioni, l'interpretazione del nome della dea è quello di "signora del cielo".

19 Quale questo sia non è purtroppo chiaro a causa del testo troppo lacunoso; cf. n. 17.

20 È questa un fatto eccezionale, perché si tratta dell'unica composizione della letteratura sumerica che ha per protagonista un animale, vd. Verderame (in stampa).

21 Per questo motivo vd. Verderame (2009).

22 È chiaro che in questo passaggio vi è un contrasto che a noi sfugge ma che all'antico lettore o ascoltatore doveva apparire evidente, come si desume dal risultato di tale operazione menzionato poche linee più in basso. Il termine sumerico umah indica un acquitrino dove possono anche vivere dei pesci, «(Enki) fece vivere uccelli e pesci presso le pozze e gli acquitrini (umah)» (Uccello e Pesce 14). Il termine sumerico qui tradotto convenzionalmente come porro (ga-rašsar) è una coltura orticola che si distingue per il fusto dritto. La pianta è spesso associata ai capelli. La dea degli inferi (Ereškigal) ha 


\section{Ocula ${ }^{23}$}

Vol 21, No 23 (July 2020) • DOI: 10.12977/ocula2020-26

Lorenzo Verderame | «Io sostituisco i cereali!». Origine e primato della palma nelle culture dell'antica Mesopotamia

Chi aveva mai visto prima una pianta come questa crescere dritta come un porro in un orto? ... Chi aveva mai visto prima un uccello come il corvo fare il lavoro di un uomo? (Inanna e Šukaletuda 66-69)

Corvo coltiva alacremente l'orto e a un certo punto si arrampica sulla palma e compie un'operazione con il kohl. Al termine, per ordine del suo signore Enki, Corvo torna nell' $a b z u^{23}$ da dove probabilmente era partito al principio del mito.

A questo punto Corvo scompare dalla scena e il protagonista diviene Šukaletuda, il giardiniere. ${ }^{24}$ Nonostante le sue attenzioni, particolarmente rivolte all’irrigazione del palmeto, gli alberi non danno frutti e Šukaletuda le abbatte. Un'improvvisa tempesta di sabbia acceca gli occhi di Šukaletuda e il protagonista ha quella che potremmo definire una visione.

[Levò gli occhi alle terre superiori] e vide i grandi [dè]i del Kur dove sorge il sole. [Levò gli occhi alle terre inferiori] e vide i [grandi dèi] del Kur dove cala il sole. [Vide un fantasma solitario che errava]. Riconobbe dai segni una divinità solitaria che errava. [Vide] qualcuno dai ME perfetti. [Guardava] (qualcuno il cui) destino (era stato stabilito) dagli dèi. ${ }^{25}$ (Inanna e Šukaletuda 101-106)

Il passo è estremamente importante e non è mai stato pienamente considerato nel suo significato e in relazione alla fine cui è destinato il protagonista. Šukaletuda sarà condannato a morte dalla dea Inanna e il passo in questione descrive una visione dell'immediato futuro del giardiniere. ${ }^{26}$ Le traduzioni proposte dall'editore del mito e seguite nella maggior parte delle antologie si allontanano dal testo sumerico traducendo le prime due linee «[Levò gli occhi verso alle (terre) basse] e vide i grandi [dè]i del paese, là dove sorge il sole. [Levò gli occhi alle (terre) alte] e vide $\mathrm{i}$ [grandi dèi] del paese, là dove cala il sole». ${ }^{27} \mathrm{Il}$ temine usato nel testo sumerico, kur, indica principalmente "montagna, paese straniero, Inferi". Gli dèi che Šukaletuda vede non sono "gli dèi del paese", ma sono gli dèi del Kur, ovvero degli Inferi. In quest'ottica diviene anche più chiaro il riferimento al sorgere e calare del sole. Infatti, sebbene il Kur-Montagna-Inferi si trovi a Oriente, quindi dove sorge il sole, esso ha un altro accesso anche a Occidente, dove tramonta il sole. È da questi due punti, infatti, che il sole entra ed esce per il suo tragitto infero durante la notte. Questo passaggio è segnato dalle due montagne gemelle Mašu sorvegliate dagli uomini-scorpione (Epopea di Gil-

i capelli a ciuffi o mazzi come ga-raš (La discesa di Inanna agli Inferi 235, 262) oppure nel cordoglio se li strappa come se fossero ga-raš (Gilgameš, Enkidu e gli Inferi A 204).

23 L'abzu è la regione cosmica delle acque dolci sotterranee, residenza e dominio del dio Enki.

24 Vd. n. 17.

25 Nonostante le lacune il passo è ricostruito in base alle linee 148-154 e 270-276 dove Šukaletuda riferisce gli eventi prima al padre e poi alla dea Inanna.

26 Diversi sono i sogni forieri di morte nella letteratura sumerica e accadica. Per il contesto e i paralleli con la vicenda di Šukaletuda, va ricordato il sogno di Dumuzi, sposo/amante della dea Inanna; per l'omonima composizione vd. Alster (1972).

27 La resa è mia ma rispetta l'interpretazione di Volk (1995: 128) seguita da eTCSL. 


\section{Ocula ${ }^{23}$}

Vol 21, No 23 (July 2020) • DOI: 10.12977/ocula2020-26

Lorenzo Verderame | «Io sostituisco i cereali!». Origine e primato della palma nelle culture dell'antica Mesopotamia

gameš IX 38-45). Il fantasma solitario ('gidim-dili) della visione può ben essere Šukaletuda,${ }^{28}$ mentre la divinità che erra e le cui prerogative divine (ME) sono perfette non può che essere Inanna. ${ }^{29}$ Va ricordato che il sumerico non distingue grammaticalmente il genere e quindi nel passo appena commentato, ma soprattutto in quello che segue, è difficile determinare se si tratti di un essere maschile o femminile. Se il riferimento alla divinità (diĝir) e alle sue "perfette prerogative" (me-šu- $\mathrm{du}_{7}-\mathrm{du}_{7}-\mathrm{da}$ ) escludono in prima istanza Šukaletuda e lasciano ampio margine all'ipotesi che si tratti di Inanna, nell'espressione successiva, "guardava (qualcuno il cui) destino (era stato stabilito) dagli dèi», il soggetto e l'oggetto dell'azione restano ambigui. È Inanna o Šukaletuda a guardare e, per contrario, è Šukaletuda o Inanna colui/colei il cui destino è stato stabilito dagli dèi?

Come per altri amanti di Inanna/Ištar e, in particolare, Dumuzi / Tammuz, Šukaletuda è un umano ${ }^{30}$ che per la relazione sessuale che ha con la dea è destinato alla morte. Come Dumuzi (Il sogno di Dumuzi), anche Šukaletuda ha una visione della sua morte. Accecato dalla tempesta di sabbia gli appaiono gli dèi inferi, sé stesso (il fantasma), e la divinità (Inanna) che ne determinerà il destino.

\subsection{L'ombroso pioppo}

Dopo la visione di Šukaletuda, il testo prosegue facendo riferimento a un altro albero $\left(\operatorname{asal}_{2}\right)$, da identificare probabilmente con il pioppo dell'Eufrate. ${ }^{31}$ Nel campo vi è una sola area ombreggiata, là dove sorge appunto il pioppo. Ed è grazie a esso che l'ombra non diminuisce mai, né al mattino né a mezzogiorno né a sera. È qui che la dea, stanca del suo girovagare, si fermerà a riposare ed è all'ombra del pioppo che Šukaletuda consumerà il suo misfatto, approfittando sessualmente della dea addormentata. È difficile dire se il riferimento al pioppo quale sola fonte di ombra nel campo sia da ricollegare al fatto che, a causa dell'infruttuosità delle palme, Šukaletuda le abbatte lasciando dunque il terreno deserto, a eccezione del pioppo. Certo l'albero isolato potrebbe fare da pendant al fantasma e al dio solitari della visione che sono i protagonisti dell'evento scatenante che avrà luogo proprio sotto di esso..$^{32}$

28 In un'altra composizione (Inanna e Bilulu), Inanna uccide e trasforma in spiriti erranti del deserto due dei tre personaggi che ritiene colpevoli della morte di Dumuzi o di un torto verso quest'ultimo.

29 L'espressione "essere/rendere perfetto" ( $\left.\check{\mathrm{s} u ~} \mathrm{du}_{7}\right)$ in relazione con i me si trova impiegato per descrivere diverse divinità, ma tra esse distacca Inanna, non solo per la presenza costante nelle sue descrizioni e nei suoi miti dei me, ma anche perché nell'introduzione al mito qui discusso, la dea è chiaramente qualificata come "colei dai me perfetti» ovvero «colei che rende perfetti i me» (me šu $\mathrm{am}_{3}-\mathrm{du}_{7}-\mathrm{du}_{7}$, Inanna e Šukaletuda 11 // 12).

30 In tutto il testo il nome di Šukaletuda non è mai premesso dal determinativo divino. Similarmente, va ricordato che in origine Dumuzi è un essere umano che morendo viene divinizzato.

31 Vd. in generale Postgate (2004).

32 Per il pioppo quale albero solitario (dili) vd., per esempio, il passo della lamentazione Udam ki amuš l. 75, «come un pioppo solitario piantato sulla riva» (Cohen 1988: 138). 


\section{Ocula ${ }^{23}$}

Vol 21, No 23 (July 2020) • DOI: 10.12977/ocula2020-26

Lorenzo Verderame | «Io sostituisco i cereali!». Origine e primato della palma nelle culture dell'antica Mesopotamia

Il pioppo $\left(\mathrm{asal}_{2}\right)$ è sicuramente un albero importante nella tradizione sumerica. ${ }^{33}$ Associato all'Eufrate così come ai terreni paludosi che caratterizzano il sud mesopotamico, il pioppo è celebrato per l'ombra che procura, ${ }^{34}$ un aspetto centrale questo nella tradizione mesopotamica radicata in ambienti desertici dove non vi è scampo al sole e l'ombra costituisce una concreta protezione. Al passo sopra citato, altri se ne possono aggiungere. Gudea negli ampi lavori di costruzione del santuario per il dio poliade Ninĝirsu pianta dei pioppi tutto intorno perché possano proiettare la loro piacevole ombra (Gudea Cilindro $A$ xxii 18-19). Più interessante è un passo tratto da composizioni interpretate come inni o liriche amorose. I protagonisti sono Dumuzi e Inanna, che impersonano qui, non "il dio che muore" e la dea dell'inversione, ma il prototipo dei due amanti o futuri sposi. ${ }^{35}$ Nella composizione nota come Dumuzi e Inanna $R$ la dea, ansiosa di incontrare il suo futuro sposo, lo associa al «pioppo, il luogo di frescura».${ }^{36} \mathrm{La}$ frescura come metafora dell'amore, più specificamente il refrigerio quale appagamento di un desiderio, è ben nota nella tradizione delle liriche d'amore. Un'altra composizione dedicata a Dumuzi e Inanna ha come incipit proprio il riferimento all'amante che porta refrigerio, «Colui che rinfresca, colui che rinfresca» (Dumuzi e Inanna T 1). Potrebbe dunque non essere casuale la relazione tra frescura e pioppo, da una parte, e la consumazione dell'atto sessuale con Inanna di cui è protagonista Šukaletuda nel nostro mito.

\subsection{Gli alberi e le metafore della morte}

Prima di proseguire con la palma e il suo successivo coprotagonista o meglio antagonista arboreo, il tamarisco, è il caso di soffermarsi sull'associazione del pioppo e di altri alberi con la morte. Nelle lamentazioni sumeriche o bilingue del II e I millennio a.C. troviamo metafore nelle quali differenti alberi sono usati per descrivere la vita spezzata del dio Dumuzi. Ne La morte di Dumuzi troviamo citato l'albero mes, assieme al «pioppo, i cui frutti sono morti e che stende lì la sua ombra» e «(l'albero?) ... che come una palma di Dilmun, lo

33 Il pioppo (șarbatu) ha una rilevanza anche nella tradizione accadica. È nelle sue radici e tra le sue fronde che il serpente e l'aquila fanno rispettivamente i loro nidi nella storia che vede i due animali protagonisti nel mito di Etana. La Serie del pioppo è una tenzone tra Pioppo e altri alberi, tra cui martû, identificato con l'alloro o il frassino; il testo, che presenta numerose lacune, è stato recentemente edito da Jiménez (2017: 157-197). Il pioppo è ampiamente usato come materiale da costruzione o per la fabbricazione di oggetti, così come lo è nei rituali, per le fonti accadiche vd. Chicago Assyrian Dictionary Ș pp. 108-109 e Jiménez (2017). Per l'ambito sumerico da notare un passo che fa anche riferimento allo scettro del dio luna che sarebbe fatto appunto di legno di pioppo (Lugalbanda nella caverna 220); per altri usi vd. Postgate (2004).

34 «Io faccio ombra tanto al nobile che all'umile», Serie del pioppo Ic 16' (Jiménez 2017: 169).

35 Per le composizioni sumeriche vd. Sefati (1998) e Mander (2005); per le composizioni accadiche vd. il recente volume di Wasserman (2016).

36 Dumuzi e Inanna R A24 // C12'; Sefati (1998: 239-240). 


\section{Ocula ${ }^{23}$}

Vol 21, No 23 (July 2020) • DOI: 10.12977/ocula2020-26

Lorenzo Verderame | «Io sostituisco i cereali!». Origine e primato della palma nelle culture dell'antica Mesopotamia

copre come una veste».$^{37}$ Frequente è l'associazione dell'abbattere o sradicare gli alberi con l'uccisione di Dumuzi. ${ }^{38}$

Schiacciano i pioppi con il loro abbondante fogliame, schiacciano distruggendoli. ... Sradicano i pioppi con il loro abbondante fogliame, schicciano distruggendoli.

$(\text { Enemani ilu ilu })^{39}$

In una composizione bilingue tarda, troviamo una serie di metafore in cui compaiono il pioppo ildag, il tamarisco e la vite sempre in riferimento a Dumuzi morto:

Mio tamarisco, che non berrà acqua nel terreno dove è cresciuto,

la cui chioma nella steppa non dà foglie!

Mio pioppo ildag, che non finirà mai (l'acqua) del suo canale,

mio pioppo $i$., sradicato dalle radici!

Mio stelo, che non berrà acqua nel terreno dove è cresciuta!

(Edena Usagake 14-18) ${ }^{40}$

Infine, una tarda lamentazione eršemma documenta una differente storia circa l'arrivo di Nergal agli Inferi. Le diverse versioni della storia di Nergal ed Ereškigal narrano di come Nergal, dio celeste, a seguito di una mancanza verso la dea degli Inferi sia stato costretto a discendere nell'oltretomba e lì sarebbe divenuto il consorte di Ereškigal. In Nergal ed Ereškigal si sottolinea il lato romantico della vicenda: Ereškigal, vergine e solitaria, conosce l'amore tramite Nergal e gli offre un trono accanto al suo. L'Eršemma a Nergal, invece, presenta il dio come una versione di Dumuzi, ovvero come un guerriero ucciso e trascinato nell'oltretomba. Nergal, steso e inerte, è chiamato, in due strofe parallele, come «il pioppo ildag di Gilgameš». ${ }^{41}$ Per quanto l'espressione non abbia paralleli e risulti oscura è chiara l'associazione dell'albero con un altro dio infero, Gilgameš. ${ }^{42}$

\subsection{Re Palma}

Abbiamo notizia di un re Palma (lugal şiš gišimmar) menzionato in alcuni testi letterari. ${ }^{43}$ Nello specifico, il re Palma compare tra i nemici sconfitti dal

37 Kramer (1980: 10 ll. 81-83). Si noti che nelle linee successive di questa composizione si fa riferimento al corvo.

38 Il riferimento agli alberi sradicati si trova anche nelle composizioni sumeriche che celebrano la distruzione delle città della bassa Mesopotamia alla fine del III millennio a.C., anche esse chiamate lamentazioni: «Furono tagliati come palme e raccolti assieme ... Perché ci distruggono come palme ...?» (Lamentazione per la distruzione di Sumer e di Ur 195, 241).

39 Cohen (1988: 192 1. 15, 202 l. 58, 205 l. 8).

40 Cohen (1988: 691).

41 Cohen (1981: 95 ll. 35, 37).

42 Per Gilgameš quale divintà infera vd. Katz (2003: 14-17).

43 Per la palma da dattero definita "re degli alberi” vd. il passo di Palma e Vite «Io 


\section{Ocula ${ }^{23}$}

Vol 21, No 23 (July 2020) • DOI: 10.12977/ocula2020-26

Lorenzo Verderame | «Io sostituisco i cereali!». Origine e primato della palma nelle culture dell'antica Mesopotamia

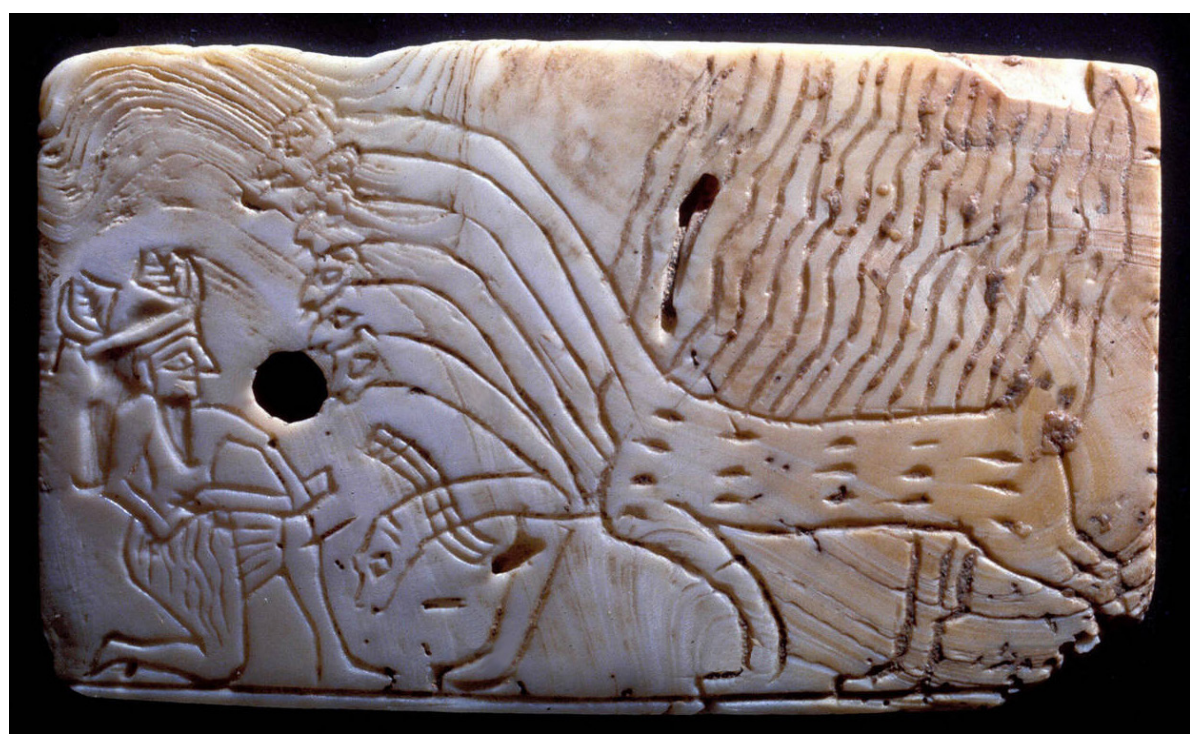

Figura 9. Placchetta con dio che combatte contro un mostro a sette teste, forse Ninurta contro il Serpente a sette teste (Bible Land Museum di Gerusalemme).

dio Ninurta/Ninĝirsu, ${ }^{44}$ ma non sappiamo nulla della vicenda in cui compariva come avversario del dio.

O Ninurta, elencherò i nomi dei guerrieri da te sconfitti: Kuli-ana, il Dragone, Gesso, il potente Rame, il bellicoso Ariete selvatico a sei teste, la barca Magilum, il signore Saman-ana, il Bisonte, il re Palma, Anzu, il serpente a sette teste. O Ninurta, tu li hai uccisi nella montagna. (Lugal-e 128-134)

Nella sala interna (del tempio di Ninĝirsu), la dove sono affisse le armi, presso la Porta della battaglia, (Gudea ha) posto l'Ariete selvatico a sei teste e ... Volto verso la città, il suo posto ammantato di aura, egli ha posto il Serpente a sette teste. In Šugalam, la sua splendida porta, ha posto il Dragone e la Palma ... (prosegue la lista delle effigi dei nemici sconfitti posti nei vari luoghi da Gudea). (Gudea Cilindro $A$ xxv 24 - xxvi 2)

Questi sono per lo più esseri caotici che vivono sulla montagna/Kur o da essi sono stati generati. ${ }^{45}$ Molti di essi, una volta sconfitti dal dio ne divengono servi-assistenti. In altri casi, invece, il dio opera un'azione civilizzatrice, trasformando elementi inerti della periferia caotica (Kur) in materie prime funzionali per le città mesopotamiche. ${ }^{46}$ È questo il senso della lunga composizione nota come Lugal-e, che narra la battaglia di Ninurta contro Asakku

sono superiore a tutti gli alberi e sono chiamato con il nome di re!» (II 26) e il passo della Serie del pioppo (II 17') citato in n. 54.

44 Heimpel (1996).

45 I due più importanti e noti, in quanto protagonisti (o meglio antagonisti) di due importanti composizioni, sono Asag/Asakku (Lugal-e) e Anzu (Anzu).

46 Vd. Verderame (2011: 116-118). 


\section{Ocula ${ }^{23}$}

Vol 21, No 23 (July 2020) • DOI: 10.12977/ocula2020-26

Lorenzo Verderame | «Io sostituisco i cereali!». Origine e primato della palma nelle culture dell'antica Mesopotamia

sulla montagna. Le pietre della montagna si schierano a favore o contro il dio e, quando questo alla fine riesce vittorioso, stabilisce il destino delle pietre, positivo per i suoi alleati, negativo per chi si è schierato con Asakku. Il destino stabilito non è altro che l'uso o la funzione che ciascuna pietra avrà nella vita quotidiana e quindi nell'artigianato. Dobbiamo immaginare quindi che nemici come Palma, ma anche Gesso e Rame, una volta sconfitti siano divenuti elementi e materie funzionali all'economia mesopotamica. In tal senso, il riferimento al re Palma potrebbe testimoniare l'esistenza di un mito oramai perduto dove si narrava una differente storia sull'origine o "domesticazione" della palma rispetto a quella presentata in Inanna e Šukaletuda.

\section{Palma e Tamarisco}

In un inciso di Inanna e Šukaletuda si descrivono le caratteristiche e gli usi delle diverse parti della palma:

La sua ... è davvero il cuore di palma, le sue foglie seccate sono davvero materiale per l'intreccio, le sue infiorescenze sono della corda dell'agrimensore - sono degne del campo del re, le sue fronde sono usate per spazzare nel palazzo del re, i suoi datteri sono posti a lato dell'orzo spulato - sono degni dei templi dei grandi dèi. (Inanna e Šukaletuda 78-84)

La palma dunque offre, oltre al prezioso frutto (datteri), materiali per gli scopi più diversi: le foglie sono intrecciate per fare ceste e stuoie, le infiorescenze per fare corde, le fronde per spazzare. ${ }^{47}$ Più dettagliata è la descrizione dei vari usi della palma in un'altra composizione, Palma e Tamarisco. Si tratta di una tenzone, una tipica composizione ben nota nella tradizione sumeri$\mathrm{ca}^{48}$ in cui sono contrapposti due principi o elementi come Zappa e Aratro, Grano e Pecora, Estate e Inverno, etc. Palma e Tamarisco è, tuttavia, nota principalmente da copie in lingua accadica ${ }^{49} \mathrm{e}$ da un solo frammento in lingua sumerica..$^{50}$

47 Vd. in generale Dowson (1921) e, per il lessico tecnico sumerico e accadico, Landsberger (1967).

48 Queste composizioni sono definite “dispute verbali” (a-da-min -du $_{11}$-ga) vd. van Dijk (1953: 31-42), Vanstiphout (1990; 1992), Ponchia (1996: 16-24; 2007).

49 Il testo è stato edito in italiano da Ponchia (1996: 67-69); più recente la revisione del testo accadico e sumerico da parte di Streck (2004); si veda anche la discussione in Jiménez (2017: 28-39), con bibliografia precedente. Di recente Jiménez (2017: 231271) ha ricostruito un'altra tenzone in accadico di cui è protagonista la palma da dattero - qui chiamata mediante l'oscuro termine arhānû - contrapposta alla vite (Palma e Vite); il testo, molto frammentario e di cui è possibile ricostruire circa cinquanta linee, è noto attraverso tre manoscritti risalenti al periodo ellenistico. Per la relazione di questa composizione e di altre con le tenzoni delle tradizioni posteriori dell'area vicinoorientale (aramaica, araba, persiana, turca), tra cui il testo medio-persiano del Draxt $\bar{\imath}$ $\bar{a}$ sūrìg ("L'albero assiro", generalmente tradotto, tuttavia, come L'albero babilonese), ma anche delle letterature greca e latina vd. la discussione in Jiménez (2017: 125-153).

50 Il frammento (Sb 12354) proviene dall'antica capitale elamita Susa ed è edito 


\section{Ocula ${ }^{23}$}

Vol 21, No 23 (July 2020) • DOI: 10.12977/ocula2020-26

Lorenzo Verderame | «Io sostituisco i cereali!». Origine e primato della palma nelle culture dell'antica Mesopotamia

La palma è sicuramente l'albero e la coltura più prestigiosa della Mesopotamia ma ha un valido rivale nel mondo vegetale. Si tratta del tamarisco,${ }^{51}$ che seppure di dimensioni modeste, costituisce nell'antica Mesopotamia il più importante albero da legname, come si evince dalle parole che i due contendenti della tenzone Palma e Tamarisco si rivolgono..$^{52}$ In un passo di un inno a Dumuzi e Inanna, le due piante sono chiaramente messe sullo stesso piano quale sinonimo di grandiosità e bellezza, quando la dea descrivendo l'avvenenza del suo futuro sposo insiste sull'acconciatura:

Il mio tesoro, il mio (amante) dalla chioma perfetta!

Il mio (amante) dalla chioma perfetta come una palma!

Il mio (amante) dal collo forte, dalla chioma perfetta come un tamarisco!

(Dumuzi e Inanna $Y$ 35-37) ${ }^{53}$

Il testo comincia con un'introduzione mitologica che rievoca gli incipit delle composizioni sumeriche: «In giorni luminosi, in notti oscure, in anni lontani» (1. 1) e prosegue con una breve cosmogonia e con l'introduzione della regalità tra gli uomini. A questo punto il re pianta nel suo palazzo una palma e un tamarisco. All'ombra del tamarisco si tengono banchetti, all'ombra della palma si riuniscono i notabili. Nonostante la gioia che i due alberi producono, presto tra loro nasce l'inimicizia e la competizione. Comincia Tamarisco declamando la sua grandezza, risponde Palma sostenendo di essere più alta $\mathrm{e}$ insistendo sulla sua superiorità legata ai datteri. Infatti, il tamarisco non porta alcun frutto, ${ }^{54}$ mentre i datteri della palma sono perfetti per la tavola del re e dei suoi notabili.

Così (rispose) Palma: «Io sono più alta di te! Ma tu, o Tamarisco, sei un albero che non fa frutti! A cosa servono i tuoi rami, o Tamarisco, senza frutti? Sono miei i frutti che [si imbandiscono] sulla tavola [del re]. Il re ne mangia e i notabili li lodano. Il frutto che io procuro al giardiniere lui lo porta alla regina. Fa crescere la madre il figlio, il grande mangia l'omaggio della mia forza. I miei frutti [stanno] costantemente davanti al re!». (Palma e Tamarisco 14-20)

da Cavigneaux (2003: 53-57). Si tratterebbe, tuttavia, non di una versione precedente quella accadica, ma probabilmente di un esercizio di traduzione dall'accadico al sumerico; vd. Cavigneaux (2003: 54 n. 7). Per uno studio sulla distribuzione dei manoscritti di quest'opera e loro circolazione vd. Jiménez (2017: 28-35).

51 Sum. šinig, acc. bīnu.

52 Si veda l'entrata del Reallexikon curata da Streck (2012), con bibliografia, e la più estesa discussione in Streck (2004: 274-286).

53 Cf. anche il passo dalla Serie del pioppo citato alla nota seguente. Per la chioma del tamarisco vd. anche il passo della lamentazione Edena usagake discusso sopra. Per un altro riferimento all'ampiezza della chioma della palma, vd. «Che la tua palma estendendo le sue fronde, come un fico estenda(?) la gioia!» (Šulgi N 93-94).

54 Il motivo dell'improduttività del tamarisco è ripreso nella Serie del pioppo, dove Frassino ribatte: «[O Piop]po, [tu non sei] carne degli dèi, come Tamarisco; [il tuo] frutto non è [commestibile], come Palma, re degli alberi!» (Serie del pioppo II 16'17’; Jiménez 2017: 174-175). 


\section{Ocula ${ }^{23}$}

Vol 21, No 23 (July 2020) • DOI: 10.12977/ocula2020-26

Lorenzo Verderame | «Io sostituisco i cereali!». Origine e primato della palma nelle culture dell'antica Mesopotamia

Tamarisco, tutt'altro che intimidito, oppone al frutto della palma il valore del suo legno e argomenta la sua superiorità sostenendo che come una schiava verso la padrona, Palma porta il suo frutto al Tamarisco (tavola) «perché sia piacevole e gradito» (1. 24). La risposta di Palma è purtroppo in lacuna e conosciamo solo la successiva argomentazione di Tamarisco che, mentre nel passo precedente aveva elogiato il suo legno, ora vanta gli oggetti che da esso si ottengono, in particolare tutti gli utensili del palazzo (tavola, coppa, scodella, madia, telaio e spola). Inoltre, aggiunge che è lui la materia e lo strumento principale della pratica esorcistica («Io sono l'esorcista e purifico la casa del dio» 1.36$) .55$

Palma replica elogiando il suo ruolo nelle funzioni religiose. In particolare, suo è il rito di purificazione (šuluhhu) ai quattro venti e nelle celebrazioni le sue fronde coprono il pavimento. Palma, tuttavia, contrattacca l'avversario rimarcando l'uso umile che se ne fa quale ricettacolo di immondizia invece che di datteri.

Palma aprì la bocca e rispose dicendo a suo fratello: «Nel gizinakku, ${ }^{56}$ durante le celebrazioni per il principe Sîn, senza di me il re non celebra i riti. I riti di purificazione ai quattro venti sono i miei riti di purificazione e le mie fronde sono sparse a terra quando si fanno le feste. In quei giorni, il tamarisco è condotto nella mano del birraio e su di lui si ammonticchia la feccia (della birra) invece che pile di datteri!». (Palma e Tamarisco $37^{\prime}-42^{\prime}$ )

Tamarisco risponde esaltando il profumo del suo legno e delle sue essenze che si sprigiona nei quartieri (città) degli artigiani o nelle fumigazioni rituali. La palma, invece, «è nelle mani del macellaio» e le sue fronde sono imbrattate di sangue ed escrementi, ovvero sono usate per coprire il pavimento e ripulirlo dei resti della macellazione degli animali.

La risposta di Palma riprende l'argomento di Tamarisco. Come quest'ultimo aveva invitato Palma ad andare a vedere quello che succedeva nella città dei "suoi" artigiani, Palma invita Tamarisco ad andare a vedere cosa succede nella città della "sua" festa, dove gli artigiani, il mezzo stesso dell'esaltazione di Tamarisco, venerano Palma.

Rispose Palma dicendo a suo fratello: «Vieni, andiamo, io e te, alla città della mia festa, il luogo di gioia. Il tuo artigiano, o Tamarisco, il falegname carico sulla nervatura e il carpentiere mi loda e mi venera quotidianamente!». (Palma e Tamarisco 50'-52')

Tamarisco si affretta a replicare come sia lui ad arrecare ogni strumento utile per l'agricoltura. Dai suoi rami si fa la zappa, lo strumento con cui si compiono i principali lavori agricoli. È tramite lui che il campo coltivato produce i cereali, che sono il sostegno della regalità e il vigore della gente.

55 Per i prodotti ottenuti dal tamarisco e il suo uso nell'esorcistica vd. Streck (2004: 276-278, 282-285; 2012).

56 Il luogo delle offerte per il dio luna Sîn. 


\section{Ocula ${ }^{23}$}

Vol 21, No 23 (July 2020) • DOI: 10.12977/ocula2020-26

Lorenzo Verderame | «Io sostituisco i cereali!». Origine e primato della palma nelle culture dell'antica Mesopotamia

Palma similarmente ribatte che è lei, invece, a fornire i principali strumenti per l'agricoltura: dalle sue foglie, dalla sua nervatura e dalle sue fibre si ottengono corde, finimenti, fruste. ${ }^{57}$

Aprì ancora la bocca e rispose Palma dicendo a suo fratello, Tamarisco: «Io più di te sono utile alla gente! L'agricoltore provvedo di ogni cosa utile: la cavezza, la frusta, i finimenti per il giogo, la fune ritorta per il lavoro assegnato, le redini del carro [...] Qualsiasi cosa io fornisco all'agricoltore. Io sono superiore a te!». (Palma e Tamarisco 60 '-63')

Le fonti principali si arrestano qui e un unico frammento prosegue con un'ulteriore serie di botta e risposta. Tamarisco a questo punto elogia la sua grande forza. Per quanto il passo sia frammentario, è chiaro che Tamarisco insiste sulla qualità del suo tronco comparato al tronco o alle fronde della palma che sono facilmente tagliate «come colui che lavora le canne» (Ab l. 42').58

La replica di Palma insiste su un argomento differente: il dattero può sostituire l'orzo e permette a tutta la gente, soprattutto gli indigenti, di sopravvivere fino a tre mesi cibandosi solo di esso.

(Rispose Palma:) «Io ti sono superiore sei volte, sette volte [...]! Io sostituisco i cereali! Per tre mesi [...] l'orfano, la vedova, il povero [...] possono cibarsi del mio cibo che non manca: dolci datteri [...]». (Palma e Tamarisco Ab 45'-50')

A questo punto tutti i testimoni a nostra disposizione si interrompono. Non conosciamo chi sia il vincitore tra i due antagonisti in questa contesa. Nelle tenzoni sumeriche si raggiunge un impasse per cui è chiamato a intervenire quale giudice un dio o un sovrano. La risoluzione non sempre è una logica conseguenza delle argomentazioni dei due contendenti e nella decisione del giudice spesso intervengono fattori di natura teologica. Apparentemente nel nostro caso è Palma a prevalere su Tamarisco. È quest'ultimo che introduce argomenti cui Palma puntualmente ribatte mostrando la sua superiorità. È Tamarisco il primo a parlare e (1) iniziare la tenzone, vantando la sua grandezza. Palma risponde di essere più alta e denigra Tamarisco per l'infruttuosità dei suoi rami esaltando, invece, il valore del suo frutto (dattero). (2) Tamarisco allora introduce un differente argomento, il valore del suo legno. La risposta di Palma è perduta. (3) Tamarisco sottolinea come tutti gli utensili del palazzo siano fatti con il suo legno e il fatto che lui sia la materia principale dell'esorcista. Palma ribatte esaltando la sua funzione nelle celebrazioni religiose. (4) $\mathrm{Al}$ vanto di Tamarisco circa il profumo del suo legno nella "città" degli artigiani, Palma ribatte esaltando come gli stessi artigiani si piegano a lei nella "città" della festa. (5) Di nuovo, al vanto di Tamarisco di costituire il materiale della zappa, strumento principale dell'agricoltura, Palma replica citando i differenti usi che si fanno delle sue parti. (6') Nell'ultima parte, conservata frammenta-

57 Un passo in un inno alla dea Nisaba (Nisaba A 48-49), menziona un'arma o bastone (šita) fatto di palma che Enki usa per colpire le vesti.

58 Per il motivo delle palme abbattute vd. n. 38. 


\section{Ocula ${ }^{23}$}

Vol 21, No 23 (July 2020) • DOI: 10.12977/ocula2020-26

Lorenzo Verderame | «Io sostituisco i cereali!». Origine e primato della palma nelle culture dell'antica Mesopotamia

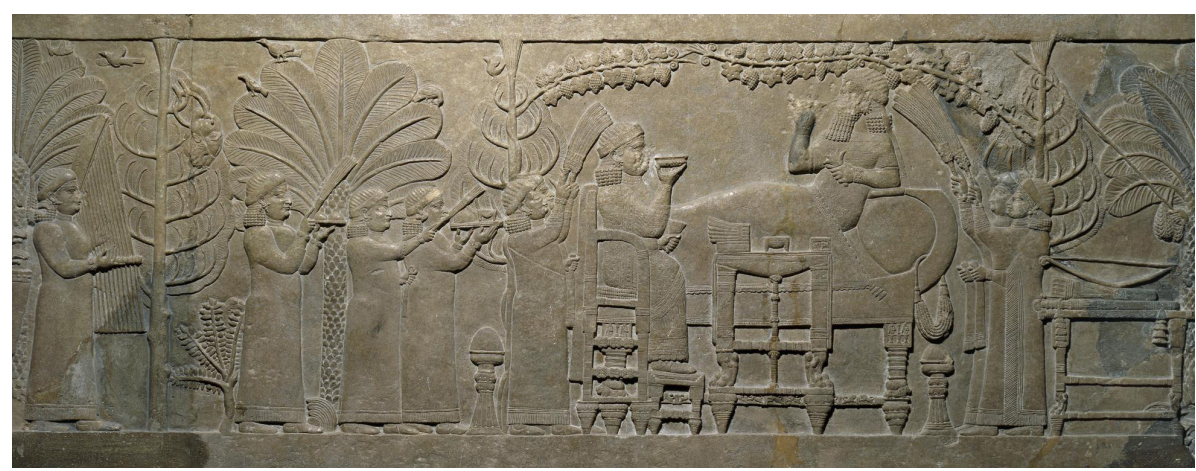

Figura 10. Rilievo assiro con la scena del cosiddetto banchetto di Assurbanipal, sullo sfondo si distinguono palme, viti e quello che potrebbe essere identificato come l'albero eren (British Museum, BM 124920).

riamente, Tamarisco esalta la qualità del suo tronco e Palma ribatte esaltando la capacità del dattero di sostituire i cereali. A ciò si aggiunge l'estrema importanza della palma nella cultura sumerica e il suo epiteto di "re degli alberi", ma, di nuovo, va, tuttavia, sottolineato che questa è la nostra percezione e la conclusione poteva essere del tutto differente.

In sintesi, la successione degli argomenti di Palma sono (1) nobiltà del frutto, (2) [...], (3-4) centralità nelle funzioni religiose, (5) utilità quale materia prima, (6') primato del dattero che può sostituire i cereali. Oltre agli aspetti pratici (intreccio delle foglie e delle fibre) Palma insiste sulla sua relazione con la regalità e le sue funzioni religiose. Entrambi gli alberi sono piantati nel palazzo del re, prima la palma, poi il tamarisco. Nel primo scambio (1), Palma sottolinea come i suoi frutti siano serviti alla tavola del re, che ne mangia assieme alla corte, e come alla regina ne faccia dono il giardiniere. Nella terza e quarta parte, alla funzione nell'artigianato cui punta Tamarisco, Palma oppone il suo ruolo nelle celebrazioni religiose. Non sono solo i passi letterari a confermare questa rilevanza della palma nella sfera religiosa. Nei testi amministrativi i datteri sono la principale uscita, dopo gli animali per i sacrifici, nelle registrazioni delle uscite per le offerte rituali.

\subsection{Varietà delle immagini vegetali}

Sebbene nell'antica Mesopotamia la palma occupi un posto di rilievo tra i rappresentanti del mondo vegetale, non si tratta dell'unico albero che spicchi per rilevanza e che sia fatto oggetto di idealizzazioni e associazioni di vario tipo. Altri alberi economicamente e culturalmente rilevanti compaiono assieme alla palma o ne contendono il primato. La tenzone Palma e Tamarisco contrappone direttamente il tamarisco alla palma. Il pioppo fa la sua comparsa in Inanna e Šukaletuda ed è protagonista di una tenzone accadica che lo contrappone al frassino. Altri alberi sono menzionati in letteratura e tra questi è il caso di ricordare gli alberi mes e halub. Un passo di un inno celebrativo del secondo re della III dinastia di Ur (XXI sec. a.C.), Šulgi, ci dà un piccolo esempio della varietà delle immagini ispirate dal mondo vegetale: 


\section{Ocula ${ }^{23}$}

Vol 21, No 23 (July 2020) • DOI: 10.12977/ocula2020-26

Lorenzo Verderame | «Io sostituisco i cereali!». Origine e primato della palma nelle culture dell'antica Mesopotamia

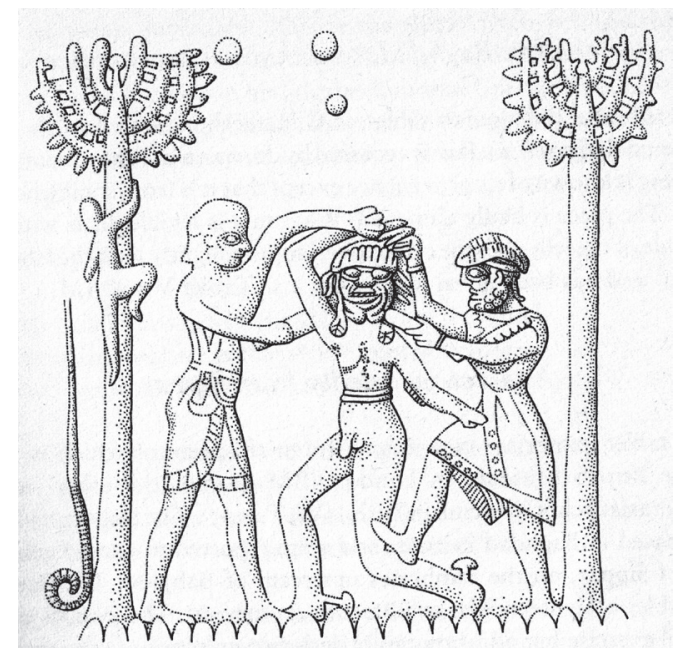

Figura 11. Terracotta da Larsa con scena dell'uccisione di Huwawa, custode della Foresta dei "cedri”, da parte di Gilgameš e Enkidu (Iraq Museum).

(O Šulgi) tu sei forte come un pioppo ildag che si erge a lato del canale, tu sei meraviglioso a vedersi come un puro albero mes dai frutti colorati, tu sei colui che vezzeggia Ninegala come una sacra palma di Dilmun, tu sei un'ombra piacevole come un albero eren ... tra i cipressi. (ك̌ulgi D 32-35)

\subsection{L'esotico eren}

Eccezionale è, invece, il caso dell'albero eren. Identificato tradizionalmente con il cedro, ${ }^{59}$ l'eren non è un albero autoctono della Mesopotamia, ma cresce nella "foresta" che si trova a Oriente, sorvegliata dal custode Huwawa (fig. 11).

È un albero dall'alto valore simbolico, ampiamente menzionato sia nei testi mitologici che nelle iscrizioni reali. Tuttavia, dell'eren si celebra l'odoroso legno con cui si costruiscono portali o si decorano templi, quindi la materia esotica importata, piuttosto che l'albero le cui caratteristiche sono poco note e che, comunque, caratterizza un paesaggio "altro", il Kur-Montagna-Inferi, con tutti i valori simbolici che ciò potrebbe comportare.

\section{Conclusioni}

Tutti gli alberi menzionati hanno associazioni simboliche con le sfere identificate per la palma e a essa spesso si sovrappongono. È questo il caso della regalità o della religione. Basti qui citare il caso dell'albero mes con cui sono composti diversi nomi di divinità e di re, primi fra tutti Gilgameš, il cui nome sumerico è Bilgames. La stessa dea Inanna, protagonista di una delle due composizioni qui analizzate, non ha un rapporto preferenziale con la palma e

59 Se il termine accadico erennu delle fonti più tarde può essere associato al cedro del Libano, diverse sono le evidenze contro l'associazione dell'eren sumerico del III millennio con quest'albero, prime fra tutte la sua localizzazione a Oriente, sull'altopiano iranico, e il riferimento alla resina (il cedro non produce resina). 


\section{Ocula ${ }^{23}$}

Vol 21, No 23 (July 2020) • DOI: 10.12977/ocula2020-26

Lorenzo Verderame | «Io sostituisco i cereali!». Origine e primato della palma nelle culture dell'antica Mesopotamia

nelle sue vicende compaiono altri alberi, come il pioppo summenzionato o l'albero halub che la dea raccoglie e pianta impropriamente a Uruk in Gilgameš, Enkidu e gli Inferi. Va inoltre ricordato che il simbolo di Inanna è la rosetta.

Tuttavia, su quest'ampio repertorio arboreo, la palma continua a godere di uno status speciale e privilegiato. Ciò è da attribuire alla sua diffusione e rilevanza economica nel sud mesopotamico, ma un ruolo centrale è svolto non solo dall'albero, ma soprattutto dal suo frutto, il dattero. Infatti, rispetto agli altri alberi su discussi, la palma è l'unico a produrre frutti, i datteri, e questi sono sovente equiparati ai cereali ovvero sono posti sullo stesso piano della principale coltura mesopotamica. Nella tradizione letteraria cuneiforme dell'antica Mesopotamia la metafora vegetale è ampiamente sviluppata e copre ambiti ben definiti. È lo stesso sistema di scrittura cuneiforme, logo-sillabografico, a favorire un gioco di associazioni e metafore che si mantiene e arricchisce durante i secoli. È interessante rilevare come una tradizione così legata all'origine dello stato sedentario e all'agricoltura non abbia sviluppato oltre un certo livello una simbologia legata alle principali colture di sussistenza (cereali e sesamo), ma che invece abbia enfatizzato una coltura che, in un ambiente peculiare come il sud mesopotamico, dove si contrappone l'eccesso di acqua (paludi) e la sua totale assenza (deserto), hanno bisogno di più cure. Si potrebbe dire che il valore della palma è direttamente proporzionale all'investimento di energie, acqua e competenze tecniche.

\section{Bibliografia}

Alster, Bendt

1972 Dumuzi's Dream. Aspects of Oral Poetry in a Sumerian Myth, Copenhagen, Akademisk Forlag.

Besnier, Marie-Françoise

2002 Temptation's Garden: The Gardener, a Mediator Who Plays an Ambiguous Part, in Parpola, S. e Whiting, R.M. (a cura di), Sex and Gender in the Ancient Near East. Proceedings of the 47th Rencontre Assyriologique Internationale, Helsinki, July 2-6, 2001, Helsinki, Neo-Assyrian Text Corpus Project.

Bottéro, Jean; Kramer, Samuel Noah

1992 Uomini e dèi della Mesopotamia. Alle origini della mitologia, Torino, Einaudi.

Brunner, Christopher J.

1980 The Fable of the Babylonian Tree. Part I: Introduction, "Journal of Near Eastern Studies", 39, 1980, pp. 191-202.

Castellino, Giorgio R.

1977 Testi sumerici e accadici, Torino, UTET.

Cavigneaux, Antoine

2003 Fragments littéraires susiens, in Sallaberger, W. et al. (a cura di), Literatur, Politik und Recht in Mesopotamien. Festschrift für Claus Wilcke, Wiesbaden, Harrassowitz. 


\section{Ocula ${ }^{23}$}

Vol 21, No 23 (July 2020) • DOI: 10.12977/ocula2020-26

Lorenzo Verderame | «Io sostituisco i cereali!». Origine e primato della palma nelle culture dell'antica Mesopotamia

Cohen, Mark E.

1981 Sumerian Hymnology: the Eršemma, Cincinnati, Hebrew Union College.

1988 The Canonical Lamentations of Ancient Mesopotamia, Potomac, CDL.

Coquerillat, Denise

1967 Aperçus sur la phéniciculture en Babylonie à l'époque de la Ière dynastie de Babylone, "Journal of the Economic and Social History of the Orient", 10, 1967, pp. 161-223.

Danthine, Hélène

1937 Le palmier-dattier et les arbres sacrés dans l'iconographie de l'Asie occidentale ancienne, Paris, Paul Geuthner.

van Dijk, Jan J. A.

1953 La sagesse suméro-accadienne, Leiden, Brill.

Dowson, V. H. W

1921 Dates and Date Cultivation Of Iraq, I, Cambridge, Heffer \& Sons.

Fuchs, Andreas

1994 Die Inschriften Sargons II. aus Khorsabad, Göttingen, Cuvillier.

Galter, Hannes D.

1989 Paradies und Palmentod. Ökologische Aspekte im Weltbild der assyrischen Könige, in Scholz, B. (a cura di), Der orientalische Mensch und seine Beziehungen zur Umwelt. Beiträge zum 2. Grazer Morgenländischen Symposium (2.-5. März 1989), Graz, RM-Druck- und Verlagsgesellschaft $\mathrm{mbH}$.

Giovino, Mariana

2007 The Assyrian Sacred Tree: A History of Interpretations, Fribourg / Göttingen, Academic Press / Vandenhoeck \& Ruprecht.

Heimpel, Wolfgang

1996 The Gates of the Eninnu, "Journal of Cuneiform Studies", 48, 1996, pp. 17-29.

Howard-Carter, Theresa

1982 An Interpretation of the Sculptural Decoration of the Second Millennium Temple at Tell al-Rimah, "Iraq", 45, 1982, pp. 64-72.

Jacobsen, Thorkild

1957 Early Political Development in Mesopotamia, "Zeitschrift für Assyriologie", 52, 1957, pp. 91-140.

Jiménez, Enrique

2017 The Babylonian Disputation Poems, Leiden - Boston, Brill.

Kalla, Gábor

2018 Date Palms, Deer/Gazelles and Birds in Ancient Mesopotamia and Early Byzantine Syria: A Christian Iconographic Scheme and Its Sources in the Ancient Orient, in Bács, T. A. et al. (a cura di), Across the Mediterranean - Along the Nile: Studies in Egyptology, Nubiology and Late Antiquity Dedicated to László Török on the Occasion of His 75th Birthday, vol. 2, Budapest, Institute of Archaeology, Research Centre for the Humanities, Hungarian Academy of Sciences and Museum of Fine Arts. 


\section{Dcula ${ }^{23}$}

Vol 21, No 23 (July 2020) • DOI: 10.12977/ocula2020-26

Lorenzo Verderame | «Io sostituisco i cereali!». Origine e primato della palma nelle culture dell'antica Mesopotamia

Katz, Dina

2003 The Image of the Netherworld in the Sumerian Sources, Bethesda, CDL.

Kramer, Samuel Noah

1980 The Death of Dumuzi: A New Sumerian Version, “Anatolian Studies”, 30, 1980, pp. 5-13.

Landsberger, Benno

1967 The Date Palm and its By-products according to the Cuneiform Sources, Graz, im Selbstverlage des Herausgebers.

Mander, Pietro

2005 Canti sumerici d'amore e morte. La vicenda della dea Inanna/Ishtar e del dio Dumuzi/Tammuz, Brescia, Paideia.

Mařik, Tomáš

2003 Sex, Religion and Antimony. Zu einer apolitischen und ahistorischen Deutung von Inana und Šukaletuda, "Wiener Zeitschrift für die Kunde des Morgenlandes”, 93, 2003, pp. 147-166.

Michel-Dansac, Fanny; Caubet, Annie

2013 L'iconographie et le symbolisme du palmier dattier dans l'Antiquité (ProcheOrient, Égypte, Méditerranée orientale), "Revue d'ethnoécologie”, 4, 2018, $<$ http://journals.openedition.org/ethnoecologie/1275>.

Moorey, Peter R. S.

1999 Ancient Mesopotamian Materials and Industries: The Archaeological Evidence, Winona Lake, Eisenbrauns.

Pettinato, Giovanni

2001 Mitologia sumerica, Torino, UTET.

Ponchia, Simonetta

1996 La Palma e il tamarisco e altri dialoghi mesopotamici, Venezia, Marsilio.

2007 Debates and Rhetoric in Sumer, in Dascal, M. e Chang, H. (a cura di), Traditions of Controversy, Amsterdam - Philadelphia, John Benjamins Publishing Company.

Postgate, John N.

2004 Pappel, in Streck, M. P. (a cura di), Reallexikon der Assyriologie, X/5-6, Berlin - Boston, De Gruyter.

Rendu Loisel, Anne-Caroline

2013 Heurs et malheurs du jardinier dans la littérature sumérienne, in Barbu, D. et al. (a cura di), Mondes Clos: Cultures et jardins, Gollion, Infolio.

Sefati, Yitschak

1998 Love Songs in Sumerian Literature. Critical Edition of the Dumuzi-Inanna Songs, Ramat-Gan, Bar-Ilan University Press.

Seidl, Ursula; Sallaberger, Walther

2006 Der Heilige Baum, “Archiv für Orientforschung”, 51, 2005/o6, pp. 54-74. 


\section{Ocula ${ }^{23}$}

Vol 21, No 23 (July 2020) • DOI: 10.12977/ocula2020-26

Lorenzo Verderame | «Io sostituisco i cereali!». Origine e primato della palma nelle culture dell'antica Mesopotamia

Selz, Gebhard J.

2001 Sex, Crime, and Politics. Zur Interpretation sumerischer Literaturwerke. Überlegungen zu Inana-k und Šukaletuda, "Journal of Ancient Civilizations”, 16, 2001, pp. 37-58.

Streck, Michael P.

2004 Dattelpalme und Tamariske in Mesopotamien nach dem akkadischen Streitgespräch, "Zeitschrift für Assyriologie", 94, 2004, pp. 250-90.

2012 Tamariske, in Streck, M. P. (a cura di), Reallexikon der Assyriologie, XIII/56 , Berlin - Boston, De Gruyter.

Vanstiphout, Herman L. J.

1990 The Mesopotamian Debate Poems. A General Presentation, I, “Acta Sumerologica”, 12, 1990, pp. 271-318.

1992 The Mesopotamian Debate Poems. A General Presentation, II, "Acta Sumerologica”, 14, 1992, pp. 339-367.

Verderame, Lorenzo

2009 La vestizione di Inanna, in Botta, S. (a cura di), Abiti, corpi, identità. Significati e valenze profonde del vestire, Firenze, Società Editrice Fiorentina.

2011 L’immagine della città nella letteratura sumerica, in Dolce, R. e Pellitteri, A. (a cura di), Città nel Vicino Oriente e nel Mediterraneo. Linee di storie e di simboli dall'antichità ad oggi, Palermo, Flaccovio.

2016 Letterature dell'antica Mesopotamia, Firenze / Milano, Le Monnier / Mondadori Education.

2017 Introduzione alle culture dell'antica Mesopotamia, Firenze / Milano, Le Monnier / Mondadori Education.

[in stampa] Animal agents in Sumerian literature, in Recht, L. e Tsouparopoulou, C. (a cura di), Fierce lions, Cambridge, McDonald Institute.

Volk, Konrad

1995 Inanna und Šukaletuda. Zur historisch-politischen Literaturwerkes, Wiesbaden, Harrassowitz.

2004 Palme, in Edzard, D. O. e Streck, M. P. (a cura di), Reallexikon der Assyriologie, $X / 3-4$, Berlin - New York, De Gruyter.

Wasserman, Nathan

2016 Akkadian Love Literature of the Third and Second Millennium BCE, Wiesbaden, Harrassowitz.

Zabar, Ahmed F.; Borowy, Andrzey

2012 Cultivation of date palm in Iraq, "Annales Universitatis Mariae CurieSkłodowska Lublin - Polonia, sec. EEE”, XXII/1, 2012, pp. 39-54.

Lorenzo Verderame è Professore associato di Assiriologia presso la "Sapienza" Università di Roma, dove insegna Lingua e letteratura sumerica e accadica. È autore di numerose monografie e articoli dedicati a differenti aspetti delle culture dell'antica Mesopotamia, tra cui l'editio princeps dei primi sei capitoli del compendio astrologico Enūma Anu Enlil (2002) e cinque volumi con l'edizione di documenti neo-sumerici. Le sue ricerche si sono concentrate sull'amministrazione e la cultura materiale della Mesopotamia del III millennio, sulle fonti documentarie del periodo neo-assiro e, più in generale, su vari aspetti delle religioni e delle letterature dell'antica Mesopotamia. Svolge attività di ricerca e collabora con diverse istituzioni universitarie e musei, tra 


\section{Dcula ${ }^{23}$}

Vol 21, No 23 (July 2020) • DOI: 10.12977/ocula2020-26

Lorenzo Verderame | «Io sostituisco i cereali!». Origine e primato della palma nelle culture dell'antica Mesopotamia

i quali il British Museum (Edizione dei testi neo-sumerici da Umma; Ur digitisation project) e la Yale Babylonian Collection dove dirige il progetto di edizione delle lettere paleo-babilonesi. Dal 2014 è l'epigrafista della missione archeologica italiana a Nina (Tell Surghul) nell'Iraq meridionale. 